\title{
Educación para la Ciudadanía en Chile
}

\author{
María G. Olivo \\ Universidad Católica del Maule, Escuela de Trabajo Social, Campus Nuestra Señora del Carmen, Carmen \\ 684, Curicó - Chile (e-mail: molivo@ucm.cl)
}

Recibido Feb. 22, 2017; Aceptado Abr. 20, 2017; Versión final Jun. 19, 2017, Publicado Oct. 2017

\begin{abstract}
Resumen
El artículo aborda los procesos de participación escolar en Chile y análisis de escenarios de educación ciudadana en liceos municipales y subvencionados buscando como objetivo caracterizar significados de ciudadanía, que hacen sentido a dirigentes estudiantiles de la VI Región de Chile. Basado en el paradigma interpretativo, se trabajó con el diseño fenomenológico para comprender las prácticas educativas y como aportan al desarrollo de ciudadanía desde la visión de nueve dirigentes estudiantiles de liceos secundarios, quienes relatan prácticas cotidianas y significados de su experiencia estudiantildirigencial. Se construyen cinco categorías de análisis: valor de la ciudadanía, concepción política de lo partidario y político, subjetividad juvenil con responsabilidad movilizadora, liceos como espacios ciudadanos, y liderazgo femenino. Todo esto da cuenta que los estudiantes secundarios, constituyen un potencial social de protagonismo que se perfila desde su propia subjetividad como ciudadano político, con responsabilidades ciudadanas y liderazgos femeninos, para su vida como adultos.
\end{abstract}

Palabras clave: ciudadanía; dirigente estudiantil; enseñanza formal; política; liderazgo

\section{Education for Citizenship in Chile}

\begin{abstract}
The article approaches the processes of school participation in Chile and the analysis of scenarios of civic education in municipal and subsidized high schools (liceos) searching as an objective of characterizing citizenship meanings that make sense to student leaders of the VI Region of Chile. Based on the interpretative paradigm, the work considers a phenomenological design to understand the educational practices and how they contribute to citizenship development from the vision of nine student leaders of secondary schools who describe daily and meaningful activities of their experience as leader students. Five analysis categories were defined: value of the citizenship, conception of politics and parties, juvenile subjectivity with active responsibility, high schools as civic spaces, and feminine leadership. All this gives indication that secondary students constitute a social potential of protagonism that is created from their own subjectivity as political citizens, with civic responsibilities, and feminine leaderships, for their life as adults.
\end{abstract}

Keywords: citizenship; student leader; formal teaching; politics; leadership 


\section{INTRODUCCION}

En la década de 1990 con el retorno a la democracia, Chile ha optado por incorporarse no sólo en el ámbito económico en el concierto mundial, sino también en lo que respecta a directrices políticas y valóricas. Los avances más notables se sitúan en el ámbito del aseguramiento de la democracia como régimen político legítimo y el respeto a los Derechos Humanos como encarnación de los ideales del bien común (Francke y Ojeda, 2013). En Chile, se inicia una reforma educacional que incorpora profundos cambios curriculares y da cuenta de las necesidades de la sociedad futura (Cox, 2003). Esta reforma está impregnada de una perspectiva cultural y comenzó a implementarse de manera gradual a partir de 1997, para completarse en el año 2002. Contiene un conjunto de orientaciones valóricas denominadas Objetivos Fundamentales Transversales (OFT) y Contenidos Mínimos Obligatorios (COM), parte importante de estos objetivos están asociados a educación para una formación ciudadana; es importante destacar que la formación de ciudadanía activa, implica generar espacios de participación social-comunitaria, destacando la capacidad de liderazgo para organizar a la población en pos de objetivos, que en este escenario buscan mejorar la calidad de vida de un contexto vulnerable (Briede et al., 2016 ). Acorde a las demandas actuales, se incorpora en el currículo, de manera explícita, temas que son preocupación central de la sociedad y que se vinculan con una mirada de ciudadanía integral, destacando derechos humanos, medio ambiente, igualdad de oportunidades, igualdad de género, respeto y valoración de la diversidad cultural (PNUD, 2002).Sin embargo, en la vida cotidiana escolar, el componente de la formación ciudadana es uno de los menos comprendidos, siendo también posible observar, la inexistencia de un eje conductor que oriente la formación escolar hacia la formación de ciudadanía moderna y democrática.

En el año 2009, los países que participaron en el estudio de la International Association for the Evaluation of Educational Achievement, señalan que una educación cívica que incorpore la complejidad actual del ejercicio de la ciudadanía, debe ser multidisciplinaria, participativa, interactiva, relacionada con la vida, llevada a cabo en un ambiente no autoritario, enterada de los desafíos de la diversidad social y con la colaboración de los padres y la comunidad, además de la escuela. También es reconocido el hecho que los objetivos de la educación para la ciudadanía deben ser asumidos en todo el currículo, la vida cotidiana en la escuela, en el clima de esta y en la sala de clases (Gobierno de Chile, MINEDUC, Estudio Internacional de Educación Cívica y Formación Ciudadana, ICCS, 2010). Como antecedente relevante se señala que, Chile aparece con 483 puntos; 17 por debajo del promedio mundial (500) en la escala de conocimiento cívico y se ubica en el lugar 24 de 36 países (ICCS, 2010). Por su parte, la prueba Internacional de Educación Cívica y Ciudadana, indica que los estudiantes de $4^{\circ}$ año de Enseñanza Media en Chile, reflejan una expectativa inferior al promedio de la muestra internacional en participación esperada en actividades políticas convencionales, tales como ingresar a un partido político, informarse sobre los candidatos de cada elección y votar en elecciones generales. Un rápido panorama de la situación en Europa a través de diversos estudios refleja también, un escaso interés por participar en la vida política, especialmente en la juventud. Desde la clase política esto se interpreta como testimonio de pasividad y abandono popular para abordar las cuestiones públicas, no teniendo en cuenta que el resultado de los bajos porcentajes de incorporación está en función de los sistemas políticos vigentes (Rubio, 1990).

A la luz de esta trilogía entre jóvenes-ciudadanía-educación, surge la pregunta que se pretende responder con esta investigación y se define como: ¿Cuáles son los significados de ciudadanía estudiantil y las categorías a considerar para la formación de un sujeto ciudadano, desde la subjetividad de los dirigentes juveniles de liceos municipales y subvencionados de la VI región de Chile? relacionados con la pregunta, se plantean los objetivos 1. Caracterizar los significados desde la visión del mundo de vida que hacen sentido a los jóvenes dirigentes estudiantiles, respecto de sus propios procesos de educación ciudadana; 2. Identificar y describir distintas subjetividades de los jóvenes dirigentes sobre participación ciudadana; 3 . Identificar significados ciudadanos que vinculan a estos actores con el contexto escolar en su vida cotidiana en tanto estudiantes y 4 . Identificar la visión crítica del joven dirigente estudiantil respecto de las formas y fondo para la formación de ciudadanía.

\section{Algunos referentes teóricos}

Esta investigación busca aportar una mirada desde los jóvenes, respecto a educación ciudadana en colegios municipales, e insinuar una distinción conceptual entre ciudadanía y participación, señalando que ciudadanía es un concepto académico, epistemológico relacionado con el significado de ser sujeto social. Mientras que el concepto de participación constituye una operacionalización de la noción de ciudadanía, concepto orientado a lo práctico, lo político e influenciado por lo ideológico. (Baeza et al. 2000).

Según la investigación, Representaciones Sociales De La Noción De Ciudadanía En Jóvenes De Enseñanza Media. (Baeza et al, 2000); señalan entre sus principales hallazgos que: "En Educación Cívica el tema ciudadanía se aborda desde una perspectiva legal, restringido a contenidos de la constitución política. 
(...) Los jóvenes conceptualizan el tema de ciudadanía con una problemática relacionada a las aspiraciones, expectativas y el acceso a la información. (...) Lo anterior señala que la noción de ciudadanía para los jóvenes está en construcción y no posee cimientos concretos en la concepción de derechos, deberes y sentido de pertenencia, sobre los cuales se estructure la formación del actor social" (Baeza et al. 2000). Entre otros, estos antecedentes, motivan a que la investigación busque problematizar desde la visión juvenil, la actual formación de la escuela para la ciudadanía y proponer algunas opciones/definiciones que aporten al currículo de educación formal en Chile.

Basándose en lo recién expuesto, además en la prioridad que el sistema educacional chileno le asigna a la formación ciudadana, como un primer espacio de socialización fuera del hogar, donde tanto en la escuela y el liceo las niñas, niños, jóvenes y adultos incorporan progresivamente la conciencia del otro y de la pertenencia a una comunidad; es decir, la escuela y el liceo se conciben como un espacio primordial de socialización. Ambos son mundos privilegiados para la interacción, donde se logra dotar de conocimientos y actitudes a los actores de la comunidad, con el fin de alcanzar una adecuada convivencia democrática; esta interacción, constituye una experiencia continua de aprendizaje ciudadano, pues en ella se configuran actitudes, emociones, valores creencias, conocimientos y capacidades, tanto individuales como colectivas, que posibilitan las identidades y prácticas ciudadanas (Gobierno de Chile, MINEDUC, 2016).

Frente a este desafío, se entenderá a la educación formal, como un espacio privilegiado de divulgación del conocimiento científico entre la población general; por tanto, gran parte de la construcción de la memoria colectiva se realizaría en ella (Francke y Ojeda, 2013). Considerando ese "espacio privilegiado", la investigación busca identificar configuraciones sociales, culturales, políticas desde el mundo de vida de los jóvenes dirigentes estudiantiles, que aporten al desarrollo ciudadano de las comunidades escolares y al desarrollo humano de la sociedad chilena. Siguiendo conceptualmente a Schütz y Luckmann, el mundo de la vida es la región de la realidad en que el hombre puede intervenir y que puede modificar mientras opera en ella mediante su organismo animado (Schütz y Luckmann, 2009).

De acuerdo a la reflexión anterior, la necesidad de generar ciudadanos es una prioridad y una tarea, entre otras, desde la escuela. La pregunta que surge entonces es, ¿qué es ser ciudadano y cómo buscar los nuevos caminos a emprender por la educación ciudadana? Si bien es cierto, el término ciudadanía no es nuevo en su acepción general, ni tampoco lo es desde el mundo de la educación, el tema es, ¿a partir de qué elementos actuales, construir una conciencia de ciudadanía escolar, en este mundo cada vez más abierto, complejo y plural al que tienen acceso los y las jóvenes estudiantes?

Ante esta interrogante y de acuerdo al planteamiento de Cabrera, (2002) se establecen las siguientes dimensiones de ciudadanía:

a) Dimensión política y de justicia asociada a un status legal que exige el reconocimiento en el ciudadano de derechos y, hoy en día, también en el de responsabilidades. En este caso, hablamos de ciudadanía como status.

b) Dimensión de Naturaleza Psicológica asociada a una identidad de ciudadano que hace que se sienta parte de una colectividad con la que se identifica y se reconoce. En este caso, se habla de ciudadanía como práctica deseable o como proceso, como hoy se plantea.

Esto apunta a que la idea de que ciudadanía no solo es un reconocimiento de status, sino también un sentimiento de "ser parte de" que se construye en la vida colectiva y por medio de la participación. En concreto, el énfasis de la formación ciudadana se refiere a cómo hacer tomar conciencia de responsabilidades y compromisos, como formar ciudadanos activos cívicamente y como desarrollar y crear los espacios estructurales y culturales necesarios para la participación.

Según la autora Cortina (2010), la ciudadanía activa, se "construye sin duda a través de la participación política, pero también en empresas, hospitales, familias, escuelas, universidades, iglesias, sindicatos y medios de comunicación. En esos lugares que tienen capacidad de generar, no solo riqueza material, sino también social y moral: No sólo capital físico, sino también capital social y capital ético, sin lo que no prosperan las naciones, mucho menos la república de la humanidad." Por su parte, al referirse a conciencia ciudadana, habrá entonces que referirse al sentido del concepto conciencia (Espíndola, 2009) como la capacidad de integrar en la vida alguna de los siguientes factores:

Capacidad de verse reflejados en los demás: apertura hacia los otros, tolerancia, compasión, amor amistad a propios y extraños.

Autonomía y Proactividad: capacidad de ver la realidad como algo que puede ser transformada y no como aquello que no podemos cambiar. 
Capacidad de actuar en distintos niveles comunitarios: familia, comunidad, nación, planeta.

Generación de una visión: capacidad de generar metas en la vida, planeación estratégica, sentido del tiempo.

Niveles de emoción y racionalidad: capacidad de conciliación y equilibrio entre ambos aspectos.

Motivaciones y objetivos de la vida: jerarquización de valores, promoción del desarrollo humano y comunitario, especialmente en los valores del espíritu.

Relaciones con la naturaleza, la ecología y la belleza.

Autoestima y otras.

En la actualidad, la noción de ciudadanía se concibe como ganancia de un proceso que empieza en la educación formal (escuela) e informal (familia, amigos, medios de comunicación, ambiente social). Cortina (2010) plantea que se aprende a ser ciudadano, llegando a lo más profundo de ser sí mismo, especialmente, en el campo educativo respecto a la forma como deberán trabajarse los espacios educativos para incorporar la práctica ciudadana en una sociedad compleja. En esta lógica una auténtica ciudadanía política se hace, no se nace en ella, educar en ella es una de las tareas centrales del sistema educativo en cualquier sociedad que se proponga forjar verdaderos ciudadanos. El movimiento teórico acerca de estas complejidades, parte de la ciencia política, en un intento por ejemplificar que pasa en las sociedades occidentales contemporáneas y de la lucha por el poder.

En síntesis, es posible señalar que: (i) Devienen en la relación Ciudadano-Estado, Ciudadano-Ciudadano; (ii) Las democráticas en cambio, desde la mirada de poder oculto y explícito; (iii) Las de multiculturalismo devienen del campo de la educación; desde la identificación de las múltiples realidades existentes.

Las tres miradas exponen distintas formas de una misma problemática, que es entender si somos o no ciudadanos activos, en donde nacerá el debate desde la misma ciudadanía. La discusión entonces, está centrada en los dilemas que existen al vivir en la democracia occidental, desde la diversidad, y la educación en sí misma. En donde la educación sobre la ciudadanía se da en contextos de exclusión; para con otros grupos que conforman la sociedad (mujeres, niños, gente de color, por cuestiones religiosas), en donde el capitalismo generaría también procesos de diferenciación. Por ello, es necesario que exista una teoría ciudadana multicultural, en donde se tome en cuenta la necesidad de conocer las teorías democráticas, desde la tensión democracia-capitalismo.

Desde las instituciones de enseñanza media; área de interés de esta investigación, en el caso dirigentes estudiantiles, suele ir produciéndose un orden, una naturaleza de las relaciones, que se basa en la existencia de una autoridad (pedagógica) cuyo accionar dentro de los parámetro que marca su rol como profesor, siempre estará presente y, por lo tanto, siempre tendrá que ser tenida en cuenta en el análisis de cualquier relación de comunicación dentro de la sala de clases (Muñoz y Martínez, 2015).

El interés de la investigación y dada su asociación a la educación formal, va en la relación de la sala de clases, pero también más allá se vincula con la activación de la vida cotidiana de representación estudiantil, impulsada por los movimientos sociales actuales, por la formación escolar y por el contexto socio-político que le corresponde al joven en la actualidad, esto "implica una ampliación del concepto de ciudadanía, dotándolo de una dimensión social" (Vilas, 1999). En relación a ello es que Vilas señala que, se entiende por ciudadanía la existencia política de las personas y ésta consiste en la presencia en el espacio público, hacerse visible a la luz pública mediante el uso de la palabra.

Siguiendo la idea de Vilas, de que ciudadanía es la existencia socio- política de las personas, y según lo planteado por Arendt respecto a que el discurso en el espacio público y la actuación conjunta de los ciudadanos, dan origen a lo político, se destaca la importancia de la participación activa de los ciudadanos en la constitución del espacio público a través de los procesos de deliberación democrática. Solo por medio de la creación y la defensa de espacios públicos alternativos, independientes, se puede defender la sociedad de tendencias autoritarias. (Arendt, 2009). Destacar que Arendt en relación a este punto indica que, responsabilidad colectiva es corresponsabilidad política para mantener el espacio público que garantiza procesos deliberativos de negociación de conflictos, puntos de vista diferentes y opuestos. En este sentido, nuevamente aparece la idea de que la escuela puede ser un "espacio privilegiado" en contra del individualismo y la privatización de lo público, en época de neoliberalismo. 
Respecto a lo anterior, recobra fuerza la idea de un clásico sobre Pedagogía Realizadora, el autor H. Giroux (1990), quién entiende y propone que la escuela, más allá de sus relaciones de poder, entre pares estudiantes, dirigentes, sin duda es un espacio público que permite promover la democracia, la convivencia a partir de las diferencias y la política.

\section{La Educación Como Pedagogía Realizadora. Breve reflexión desde Giroux}

La postura de Henry Giroux, señala que "las escuelas no son lugares neutrales y consiguientemente tampoco los profesores pueden aportar una postura neutral". Con esta perspectiva en la mente, Giroux señala que, "si los profesores han de educar a los estudiantes para ser ciudadanos activos y críticos, deberán convertirse ellos mismos en intelectuales transformativos" (Giroux, 1990).

Giroux, aporta desde la educación crítica el concepto de una práctica pedagógica que tan solo sea interdisciplinaria, transgresora y de oposición, sino que esté relacionada con un proyecto más general diseñado para promover la democracia racial, económica y política. En este sentido el autor propone integrar a la práctica pedagógica crítica, los estudios culturales, esta idea se dedica a una forma de trabajo educativo que sitúa la política en el contexto de las relaciones que articulan las representaciones simbólicas, la vida cotidiana y las tecnologías del poder, transformándose el proceso educativo en una experiencia de carácter social y del aprendizaje como resultado de diversas tareas y no como recepción pasiva de información.

A esta idea Giroux, le denomina" pedagogía realizadora" donde la teoría se busca comprender como fundamento para "intervenir en los contextos y el poder", buscando ampliar las posibilidades políticas de lo pedagógico. En este contexto, la práctica pedagógica, se convierte en realizadora porque abre un espacio en el que se discuten los límites académicos convencionales y se dan cuestiones más allá de la disciplina institucional de preguntas y respuestas; reclamando para lo pedagógico el carácter de relación de poder que interviene en la restricción de lo que estima un saber legítimo, enlazando la reflexión crítica sobre la producción de prácticas simbólicas y sociales con formas alternativas de educación democrática que ponen en primer plano las consideraciones relativas al poder y a la acción social.

Refiriéndose a la mirada política de la educación, el autor plantea que ésta permite establecer condiciones pedagógicas que hagan comprender al estudiante la forma en que el poder actúa sobre ellos, mediante ellos y para ellos. Este es una idea democrática de educación, que mira una concepción de lo político abierto y comprometido, que proporcione nuevos espacios para el trabajo comprometido con el cambio social.

\section{La Relevancia De Los Estudiantes Y Su Organización En El Proceso Educativo}

El desarrollo de la región latinoamericana pasa por el reconocimiento de valores como el desarrollo social, equidad de género, respeto por la diversidad, protección del medio ambiente, derechos humanos, derechos económicos, culturales y estos derechos serán el marco para la construcción de una ciudadanía fortalecida, en el contexto de una sociedad democrática. En este sentido, la ciudadanía en tanto ejercicio, no sólo es un derecho con miras al bienestar, sino que es la trayectoria más efectiva para garantizar que los objetivos del desarrollo estén adecuadamente representados en las decisiones públicas (PNUD, 2004).

El Informe de Desarrollo Humano en Chile (PNUD, 2000), señala como áreas estratégicas de desarrollo a) la necesidad de una sociedad fuerte, que supone la presencia de aspiraciones compartidas; b) la calidad de la vida social y las capacidades sociales aumentan con las relaciones de confianza y cooperación de las personas y, c) el Fortalecimiento de la sociedad se mide por la fuerza de la acción ciudadana como forma de expresión de la voluntad. Es decir, una fuerza mediadora entre la subjetividad de la población y el sistema político. A medida que las organizaciones estudiantiles, movimientos sociales estudiantiles, se transforman en expresiones políticas pueden inscribirse en lo que se ha venido llamando sociedad civil organizada desde el mundo estudiantil, siguiendo la idea de que la buena práctica o el ejercicio de liderazgo participativo (Araneda et al, 2016) en los centros educativos, traerá una mejora en la calidad de la educación del conjunto de los estudiantes (García y Conde, 2013).

En relación con lo anterior, entenderemos que sociedad civil son todos, pero no todos se movilizan por igual, esto se refiere, ante todo a las múltiples formas de organización y movilización. La sociedad civil no es independiente de las estructuras macro sociales, porque comprende un amplio conjunto de organizaciones, formales e informales, estas a su vez incluyen a grupos económicos, culturales, informativos y educativos de interés; de desarrollo; con una orientación específica y cívica. La sociedad civil ha tenido múltiples expresiones con relación a las fenoménicas que afectan a la sociedad, y en algunos casos han derivado en movimientos que se han transformado en verdaderos espacios de organización, en los que, se han relevado 
los conceptos de protagonismo y ciudadanía (Olivo, 2007). Estos movimientos sociales con orientación específica, han sido mundialmente conocidos.

De acuerdo a esto, es posible plantear que el elemento que está presente en las diferentes concepciones es que los componentes que configuran la ciudadanía son la base de una creación de un marco ético, para las políticas económicas y sociales orientadas al desarrollo. Asimismo, se visibiliza la trascendencia de los espacios sociales, las organizaciones como bases para gestar los valores ciudadanos.

Sin duda el espacio de formación estudiantil es fundamental en la generación de estos valores, que pasan entre otros, por una relación profesor-estudiante que pareciera hoy en día estar en desajuste; al respecto, se señala que el reconocimiento de las especificidades de la educación de jóvenes y adultos permite al profesor, partiendo de estudios teóricos repensar y cambiar la práctica (Costa, 2015). A la base de todo lo anterior, referirse brevemente al concepto de educar que significa, crear conciencia y, por consiguiente, desarrollar criterios de discernimiento, favorecer la reflexión del sentido profundo de la existencia humana. Las cualidades humanas de los educandos afectan todos los aspectos del aprendizaje; por ende, no pueden darse sólo como responsabilidades individuales (Romeo, 2001).

\section{METODOLOGIA}

Esta investigación busca caracterizar los significados desde la visión del mundo de vida que hacen sentido a los jóvenes dirigentes estudiantiles, respecto de sus propios procesos de educación ciudadana; junto con identificar las distintas subjetividades de los jóvenes dirigentes sobre su idea de participación ciudadana; que vinculen a estos actores con el contexto escolar en tanto estudiantes incorporando una visión crítica del respecto de las formas y fondo para la formación de ciudadanía estudiantil.Para los fines anteriores, el interés investigativo estuvo centrado en jóvenes estudiantes entre 17-18 años de ambos sexos, que participan en las acciones asociativas (dirigentes estudiantiles) de los Centros de Alumnos de los liceos secundarios (Municipales y Particulares Subvencionados) de la ciudad de Rancagua en la VI región de Chile.

De acuerdo a la clasificación de (Hernández et al.; 2007), en los estudios de tipo fenomenológico, donde el objetivo es analizar valores, ritos y significados de un grupo social, la muestra no probabilística de sujetos tipo es la más adecuada. En esta investigación se selecciona una muestra no probabilística o muestra intencional basada en criterios, en que la elección de los sujetos depende de la decisión del investigador; ya que no se requiere "representatividad" de elementos, sino más bien una elección de sujetos con características determinadas y donde lo que interesa es la profundidad y calidad de la información y no la cantidad ni estandarización. En este caso y de acuerdo a los tipos de muestras intencionales de la Investigación Cualitativa, se puede definir como una muestra homogénea que según la clasificación de Martínez "reduce la variación para centrarse en tópicos de gran interés para el investigador" (Martínez, 2004).

Se visitó en terreno a cada uno de los liceos de la ciudad de Rancagua, VI Región de Chile, que cumplieran con los criterios establecidos (Municipal y/o Particular Subvencionado); en cada ocasión, se informó a los cuerpos directivos de cada colegio (director o rector) sobre el sentido y contenidos de la investigación y ellos junto con autorizar el acceso a los liceos, proporcionaron datos de los posibles participantes. Posteriormente comenzó el proceso de acercamiento con los dirigentes, basándose en la referencia o información proporcionada. Se presentó buena disposición tanto de los directivos como de los dirigentes estudiantiles para participar en ambas instancias (entrevista en profundidad y grupos focal). Ambas técnicas se aplicaron en espacios proporcionados por los propios liceos.

Para la selección de estudiantes-dirigentes a entrevistar, se opta por criterios, que se describen a continuación:

Que voluntariamente deseen participar en la investigación.

Género masculino o femenino

Que estén cursando tercer o cuarto año de enseñanza media

Que tengan 17-18 años de edad

Que hayan realizado su proceso completo de enseñanza media en el liceo

Que el Dirigente estudiantil haya tenido al menos una vez participación en las instancias asociativas del liceo.

Que participen en las acciones asociativas de su liceo

Que pertenezcan a los liceos municipales y/o particulares subvencionados que voluntariamente accedieron a participar en la investigación.

La muestra final obtenida no tiene pretensiones de generalización en sus conclusiones, sino busca ofrecer resultados para promover cambios, en este caso quedo compuesta por un total de nueve dirigentes estudiantiles de establecimientos cuyas características generales se presentan en la Tabla 1: 
Tabla 1: Cuadro Características de los participantes Muestra Intencional.

\begin{tabular}{|l|l|l|l|}
\hline \multicolumn{1}{|c|}{ Tipo De Liceo } & \multicolumn{1}{|c|}{ Dependencia } & \multicolumn{1}{c|}{$\begin{array}{c}\text { Edad } \\
\text { Dirigente }\end{array}$} & \multicolumn{1}{c|}{$\begin{array}{c}\text { Género } \\
\text { Dirigente }\end{array}$} \\
\hline Técnico/Profes. & Municipal & 17 años & Femenino \\
\hline Industrial & Universidad de Santiago & 18 años & Masculino \\
\hline Industrial & $\begin{array}{l}\text { Corporación Educacional de } \\
\text { la Construcción }\end{array}$ & 17 años & Masculino \\
\hline Comercial & Municipal & 17 años & Femenino \\
\hline $\begin{array}{l}\text { Humanista } \\
\text { Científico }\end{array}$ & Municipal & 17 años & Femenino \\
\hline Comercial & Cámara de Comercio & 17 años & Femenino \\
\hline Comercial & Municipal & 18 años & Masculino \\
\hline $\begin{array}{l}\text { Humanista } \\
\text { Científico }\end{array}$ & Municipal & 17 años & Femenino \\
\hline $\begin{array}{l}\text { Humanista } \\
\text { Científico }\end{array}$ & Particular Subvencionado & 17 años & Femenino \\
\hline
\end{tabular}

Se trata de dirigentes estudiantiles que tienen entre $17-18$ años de edad, que cursan $4^{\circ}$ año de Enseñanza Media, distribuidos en 3 estudiantes de género masculino y 6 estudiantes de género femenino, habitantes de la comuna de Rancagua, VI Región de Chile. Todos ellos han desarrollado su proceso de enseñanza media en el liceo que representan. Su estrato socio - económico, se categoriza como homogéneo en términos de clase media, pertenecientes a familias cuyos padres no finalizaron sus estudios secundarios, no son profesionales y por tanto estos estudiantes pertenecen a la primera generación que finaliza su enseñanza media.

El trabajo de campo se realizó en un tiempo de dos meses, para desarrollar las entrevistas en profundidad y grupo focal, debiendo volver al campo de trabajo a completar información o profundizar en algunas categorías, cuando fue necesario (Martínez, 2004). Luego, el método de análisis se articuló sobre la comprensión de los datos y sobre el rastreo de sentido a través de la búsqueda y construcción de categorías fundamentales respecto de los hechos descritos.

\section{Procedimientos}

Se trabajó con un guion inicial, que contempló tópicos preliminares que se presentan en la Tabla 2.

\section{Validación de los datos}

La calidad de los datos se asegura apuntando a los criterios cualitativos de credibilidad y dependibilidad (Hernández et al.; 2007). Esto significa un nivel de concordancia interpretativa entre diferentes observadores del mismo fenómeno, en este sentido la confiabilidad se define a nivel interno, en la idea de que los datos deben ser contrastados a través de triangulación y/o visados por diferentes investigadores, debiendo llegar a interpretaciones coherentes (Hernández et al.; 2007).

La credibilidad, se verifica confirmando que el investigador ha captado el significado completo y profundo de las experiencias de los participantes (Hernández et al.; 2007), procediendo a la aplicación de técnicas de entrevistas en profundidad y grupo focal, así como al empleo de diversas opciones de recogida de datos para las mismas categorías, de manera tal de compensar las debilidades que pudiera tener la aplicación de alguna de ellas; garantizando que los hallazgos de la investigación estén debidamente "fundados", lo que les confiere mayor validez. Esto permitió la contrastación de los datos, detección y corrección de grados de parcialidad de cada una de las técnicas de interpretación y depuración del valor interpretativo de la información recopilada. Los antecedentes cruzados permitieron paliar las limitaciones de cada técnica. 
La dependibilidad se ratificó mediante la aplicación de técnicas de auditoría externa de pares investigadores, quienes procedieron a estudiar los datos obtenidos y los informes resultantes, siendo su condición esencial no haber participado en la recogida de datos ni en su elaboración de primer nivel en este caso, la visación de los datos se encomendó a dos investigadores colaboradores expertos.

Tabla 2: Pauta Guía Temática Para Entrevistas En Profundidad Y Grupo Focal

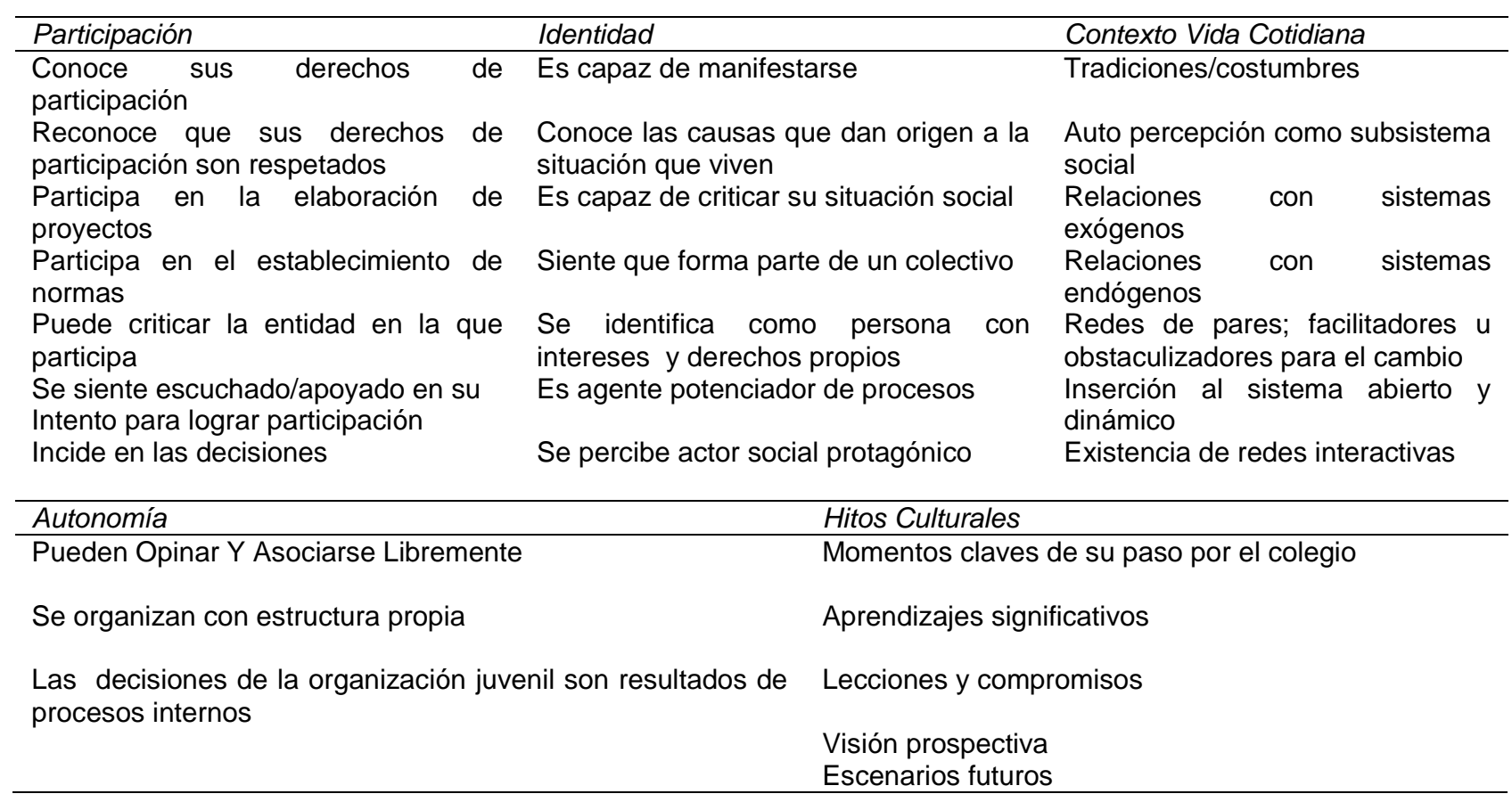

\section{RESULTADOS Y DISCUSIÓN}

A partir del análisis de las redes de significados, se logró definir las categorías y subcategorías descritas en la Tabla 3.

\section{Categoría Derechos ciudadanos. Participación Social}

En la categoría participación social; resulta clarificador establecer, que se requiere una reforma curricular a la luz de los vacíos que hoy presenta en formación ciudadana. Esta reforma debe ser orientada a tres elementos ejes que apuntan al desarrollo humano, como son la confianza, la colaboración y la cultura de paz. En este sentido, los estudiantes aun presentando resistencia con el mundo político, dado que lo asocian a la militancia en partidos, sin embargo, han sido capaces de organizarse, manifestarse, dialogar y hacer visibles situaciones vinculadas a la política educacional chilena, que permanecían en la apatía colectiva.

Partiendo de esa premisa, en materia de participación, es posible plantear que los estudiantes secundarios, constituyen un potencial social de protagonismo ciudadano que, al estar debidamente encausado en "educación en la libertad", puede ser susceptible de transformarse en un dialogante activo y permanente por parte de la sociedad civil, en diferentes temas de interés nacional, perfilándose de esta manera como un futuro ciudadano activo, una vez ingresado al mundo adulto. La ciudadanía estudiantil, aún es un proceso incipiente, donde existe un potencial de jóvenes que creen fuertemente en la democracia, pero tienen un claro rechazo y desconfianza a la política partidaria junto con no sentirse representados en esas instancias.

En la categoría liderazgo actualmente no hay fomento de la ciudadanía protagonista desde la práctica escolar. Este punto se puede fundamentar en la falta de noción por parte de los dirigentes respecto del protagonismo/liderazgo del que son portadores en el caso de que su movimiento estudiantil. Al parecer, el profesorado está más interesado en un discurso vinculado a la adaptación e integración al medio, que en gatillar procesos que transformen al estudiantado en un movimiento ciudadano, con actitud crítica y liderazgo social, que lo internalice como un interlocutor válido y permanente con la autoridad interna (en el colegio) o externa (con el estado). 
Tabla 3: Categorías de Análisis Identificadas a partir del Estudio

\begin{tabular}{|c|c|c|c|c|c|}
\hline \multicolumn{6}{|l|}{ Derechos Ciudadanos } \\
\hline Categorías & Punto A & Punto B & Punto C & Punto D & Punto $\mathrm{E}$ \\
\hline Participación Social & $\begin{array}{l}\text { Experiencias } \\
\text { positivas de } \\
\text { participación } \\
\text { estudiantil }\end{array}$ & $\begin{array}{l}\text { Ideas de } \\
\text { participación } \\
\text { estudiantil }\end{array}$ & $\begin{array}{l}\text { Participación } \\
\text { en decisiones } \\
\text { relevantes del } \\
\text { liceo }\end{array}$ & $\begin{array}{l}\text { Mecanismos } \\
\text { de } \\
\text { participación }\end{array}$ & $\begin{array}{l}\text { Obstaculizado } \\
\text { r de } \\
\text { participación } \\
\text { estudiantil }\end{array}$ \\
\hline Liderazgo & $\begin{array}{l}\text { Reconocimiento } \\
\text { de liderazgo }\end{array}$ & $\begin{array}{l}\text { Rol del líder } \\
\text { estudiantil }\end{array}$ & Perfil de líder & & \\
\hline \multicolumn{6}{|l|}{ Redes Estudiantiles } \\
\hline Política & $\begin{array}{l}\text { Concepción actual } \\
\text { del ejercicio de la } \\
\text { política }\end{array}$ & $\begin{array}{l}\text { Relación } \\
\text { estudiantes y } \\
\text { autoridades } \\
\text { políticas }\end{array}$ & $\begin{array}{l}\text { Política y vida } \\
\text { cotidiana en el } \\
\text { liceo }\end{array}$ & $\begin{array}{l}\text { Política como } \\
\text { mecanismo } \\
\text { para generar } \\
\text { cambios } \\
\text { democráticos }\end{array}$ & $\begin{array}{l}\text { Validación de } \\
\text { la política }\end{array}$ \\
\hline Movimiento estudiantil & $\begin{array}{l}\text { Visión } \\
\text { retrospectiva del } \\
\text { ME }\end{array}$ & $\begin{array}{l}\text { Relación del ME } \\
\text { con gobierno y } \\
\text { con partidos } \\
\text { políticos }\end{array}$ & $\begin{array}{l}\text { Crítica a su } \\
\text { participación } \\
\text { en el ME }\end{array}$ & $\begin{array}{l}\text { Autocritica a } \\
\text { su } \\
\text { participación } \\
\text { en el ME }\end{array}$ & $\begin{array}{l}\text { Rol de los } \\
\text { profesores en } \\
\text { el ME }\end{array}$ \\
\hline \multicolumn{6}{|c|}{ Configuraciones Del Dirigente Estudiantil } \\
\hline Aprendizajes Ciudadanos & $\begin{array}{l}\text { Aprendizajes } \\
\text { Individuales }\end{array}$ & $\begin{array}{l}\text { Desarrollo } \\
\text { Personal }\end{array}$ & $\begin{array}{l}\text { Aprendizajes } \\
\text { Colectivos }\end{array}$ & & \\
\hline Hitos & Colectivos & Individuales & Culturales & & \\
\hline \multicolumn{6}{|c|}{ Autonomía De Los Centros De Estudiantes } \\
\hline Autonomía & $\begin{array}{l}\text { Toma de } \\
\text { decisiones }\end{array}$ & $\begin{array}{l}\text { Libertad de } \\
\text { expresión }\end{array}$ & $\begin{array}{l}\text { Autoritarismo } \\
\text { de los } \\
\text { profesores }\end{array}$ & $\begin{array}{l}\text { Ausencia de } \\
\text { autonomía } \\
\text { para los } \\
\text { centros de } \\
\text { estudiantes }\end{array}$ & \\
\hline Valores & $\begin{array}{l}\text { Convivencia } \\
\text { social }\end{array}$ & Apoyo solidario & & & \\
\hline \multicolumn{6}{|l|}{ Perspectiva De Genero } \\
\hline $\begin{array}{l}\text { Reivindicación Femenina } \\
\text { en el Liderazgo }\end{array}$ & $\begin{array}{l}\text { Aprendizajes } \\
\text { individuales }\end{array}$ & $\begin{array}{l}\text { Desarrollo } \\
\text { personal }\end{array}$ & $\begin{array}{l}\text { Aprendizajes } \\
\text { colectivos }\end{array}$ & & \\
\hline
\end{tabular}

\section{Redes estudiantiles}

En la categoría política, desde los dirigentes, se percibe ausencia de un proyecto colectivo sustentable que contenga un discurso con distinciones de sujeto político. Más aún presentan una negación confusa y contradictoria frente al tema; estos jóvenes dirigentes estudiantiles, aun siendo líderes, solo se proyectan en los límites que mantienen con su colegio y con sus propios intereses individuales, mientras son estudiante; lo que se evidencia en discursos tales como "el momento más importante como Hito, es cuando ingresé a mi especialidad "por ejemplo. En este sentido, es relevante señalar que se ve aún ausente, la generación de una comunidad educativa que forma un sujeto ciudadano político.

Los dirigentes estudiantiles, consideran importante para su vida contenidos interculturales en valores que estén centrados entre otros, en la convivencia respetuosa de valores y principios ciudadanos, trato igualitario e todas las personas y la integración de los migrantes. Temas que se asemejan a lo planteado por los estudiantes chilenos desde la solidaridad, el buen trato, sin embargo, mantienen la distinción de una ciudadanía política más que intercultural, dada la naturaleza de la realidad chilena cuyo fenómeno de la inmigración en la VI región de Chile de manera incipiente recién se comienza a visualizar. De acuerdo a la investigación, existe un potencial de jóvenes que, al no estar interesados en la "oferta social y política", optan por canalizar sus vías de participación en dos tipos de actividades, por un lado, las actividades sociales benéficas, que se desarrollan a través de los centros de alumnos y por otro, lado las actividades recreativas de esparcimiento como aniversarios, campeonatos deportivos etc.

En la categoría movimiento estudianth, existe entre los dirigentes estudiantiles una diversidad de miradas frente a su participación, más allá de que todos están de acuerdo en que fue de utilidad para lograr ciertos cambios en la reformas educacionales chilenas, han planteado la sobre ideologización de algunos participantes/lideres el movimiento, teniendo en algunos casos la visión de haber sido manipulados por las instancias políticas tradicionales, ejemplificando con la "intervención de los partidos políticos en las asambleas", esta situación los deja disconformes y críticos frente a la posibilidad de futuras participaciones ya que no todos comparten los mecanismos utilizados como los largos periodos de "tomas" en los liceos, que los hizo perder clases y popularidad frente a la imagen pública del mundo adulto. 


\section{Configuraciones del dirigente estudiantil}

Categoría Aprendizajes Ciudadanos, la concepción actual del ejercicio de la política significa para los dirigentes estudiantiles, una configuración diferente a lo partidario y a "los políticos"; señalando que para la ellos la política es una herramienta de poder para el cambio en la sociedad, que permita la búsqueda de formas alternativas de ciudadanía política, ya que la actual no los representa. Para los jóvenes estudiantes el ejercicio de la democracia y la política es una opción vinculada a valores éticos más que a la necesidad de poder por poder. Hace sentido la diferencia en este caso respecto a poder y autoridad; ya que mientras la institución educacional busca la autoridad asimétrica de tipo vertical, el poder se basa en la persuasión, en el dialogo comunicativo entre pares. El poder busca la participación y no la instrumentalidad.

Categoría Hitos. Desde la subjetividad del joven estudiante en la convivencia cotidiana los principales hitos a considerar como esenciales para la formación ciudadana son: liderazgo ligado a la idea de un poder responsable y movilizador y no un "trampolín de poder"; orientación social solidaria, que no contenga diferencias de trato según género, con elementos centrados en la confianza, la colaboración y la cultura de igualdad. Claramente los estudiantes señalaron que la idea de una Educación Ciudadana Crítica aún se percibe distante debido a las prácticas asistenciales y autoritarias de los establecimientos educacionales, a una forma de participación meramente consultiva y a una relación de subordinación irreflexiva con las autoridades docentes al interior de los establecimientos.

\section{Autonomía de los centros de estudiantes}

Categoría Autonomía. Al respecto es importante recordar que una de los elementos que se plantea en el análisis y que destacan los estudiantes; se refiere a su falta de autonomía como dirigentes; están sobreprotegidos, lo que implícitamente, significa a la luz de su mirada, un desconocimiento y falta de legitimación a sus capacidades organizativas, habilidades y responsabilidades como jóvenes capaces y preparados para asumir su papel de ciudadanos; manteniendo en la actualidad, la tendencia al statu quo, o la dominación " protectora" hacia el estudiantado en la relación social estudiante -profesor.

Entendiendo que las personas se forman como ciudadanos en diversos espacios y contextos, en el plan curricular, se puede plantear de calidad, visto como transformación, donde cobra importancia la noción de cambio cualitativo, un cambio fundamental de forma, de un estado a otro producto de un proceso (Araneda et al, 2016) contempla como obligatorio la realización de proyectos de desarrollo comunitario que pongan en práctica sus experiencias y conocimientos ciudadanos y la solidaridad con otros, sin embargo lo que se hace no se considera suficiente por parte del estudiante.

Categoría Valores Un proceso de desarrollo efectivo de proyectos en terreno, es un factor que los incentiva a involucrarse en temas ciudadanos y/o contingentes como interculturalidad, envejecimiento, derechos humanos, género, apoyo solidario, que los sensibiliza con la realidad que está en el contexto social y de vida cotidiana de los estudiantes. Los jóvenes consideran importante para sus vidas contenidos interculturales en valores que estén centrados entre otros en la convivencia respetuosa de valores y principios ciudadanos, trato igualitario e todas las personas y la integración de los migrantes (Tomé et al.2010)

\section{Perspectiva de género}

En el caso de las mujeres dirigentes estudiantiles, surge una clara reivindicación por el liderazgo femenino, descalificando los liderazgos masculinos, lo que le da un carácter que se proyecta más allá del simple hecho de ser líder sino de las condiciones que éste debe tener y para ellas claramente debe ser mujer.

"yo creo que harta parte de mujeres que pueden ser líderes, pero hombres no, creo que ya el hombre tiene poca iniciativa". (Entrevistada $\left.N^{\circ} 1\right)$

También se destaca en el ámbito de género, los actuales roles societarios de la mujer y la forma como se reconoce abiertamente el fenómeno del machismo en las prácticas cotidianas del ejercicio del liderazgo, destacando la fortaleza clara, para este desarrollo, de los colegios mixtos. A este respecto cabe señalar que el análisis de la experiencia y el simbolismo que encierra el acto de subjetividad de género permite conocer e interpretar la manera como los agentes educativos permanecen atrapados en sus acciones en experiencias constantes y cotidianas de inequidad de género (Espejo, 2015) 


\section{CONCLUSIONES}

En relación con los objetivos de la investigación y consistente con sus resultados, se puede concluir los siguientes:

1) En su significado de ciudadanía los dirigentes estudiantiles, plantean la idea de "emancipación" organizativa, a través de una construcción desde espacios no formales "desde abajo" y fuera del sistema político. Ellos aspiran a ser un colectivo independiente del mundo adulto, del mundo docente y de los políticos. Cabe señalar entonces que las categorías a tener presentes para configurar una misión en la educación ciudadana son una visión ético- política, orientada al protagonismo juvenil y comprometida en una concepción de mundo donde el docente deberá ser concebido un actor reflexivo que comparte junto a otro actor y que entiende a la educación como una tarea socio/política, donde el concepto central sea el de las decisiones para la vida en sociedad y la convivencia humana. En la actualidad, los estudiantes manifiestan tener derechos de participación no vinculantes, tanto en las instancias formales como en los Consejos Estudiantiles, además de identificar una relación sobreprotectora de parte de sus profesores. Por tanto, sería interesante reconocer y avanzar formalmente en los procesos vinculantes de triestamentalidad, que permitan al dirigente validar su opinión y hacerla sentir al interior de la comunidad educativa en el ámbito de las decisiones.

2) Concepción de la política distinta a lo partidario y los políticos; respecto a los significados que desde su mundo de vida hacen sentido a los dirigentes estudiantiles, es posible concluir que una de los elementos que se plantea en el análisis y que destacan los estudiantes; se refiere a su falta de autonomía como dirigentes; están sobreprotegidos, lo que implícitamente, significaría un desconocimiento y falta de legitimación a sus capacidades asociativas/organizativas, a sus habilidades y responsabilidades como jóvenes capaces y preparados para asumir su papel de ciudadanos; manteniéndose en la actualidad, la tendencia al statu quo, o la dominación " protectora" hacia el estudiantado en la relación social estudiante profesor. En este sentido se concluye que la educación formal tiene desafíos pendientes, cual es fomentar la inquietud por los temas ciudadanos, haciendo a los jóvenes responsables de sus procesos, permitiéndoles el error y el aprendizaje, más que la sobreprotección, que es un elemento presente en la dinámica actual y altamente criticado por los dirigentes estudiantiles.

3) Desde la subjetividad del joven estudiante en la convivencia cotidiana los principales elementos a considerar como esenciales para la formación ciudadana son: a) liderazgo ligado a la idea de un poder responsable y movilizador y no un "trampolín de poder"; b) orientación social solidaria, que no contenga diferencias de trato según género, con elementos centrados en la confianza, la colaboración y la cultura de igualdad. Claramente los estudiantes señalaron que la idea de una Educación Ciudadana aún se percibe distante debido a las prácticas asistenciales y autoritarias de los establecimientos educacionales, a una forma de participación meramente consultiva y a una relación de subordinación irreflexiva con las autoridades docentes al interior de los establecimientos.

4) Liceos como espacios apolíticos. El sentido de identidad colectiva con una identidad política ciudadana, no está internalizado en el ámbito de vida cotidiana en el liceo, ya que el vínculo del estudiante con el liceo se percibe como una instancia para satisfacer su necesidad de orientación y afecto en primer lugar y, en segundo lugar, satisfacer una necesidad de tipo instrumental como futuro sujeto inserto en el sistema productivo. No hay proyecto asociativo, con la idea de ciudadano integral de deberes y derechos. Los (as) dirigentes estudiantiles respecto de la política, construyen identidades bajo su lógica generacional, no significa que carezcan de visión política, pero al percibir que son vistos y tratados de manera instrumental, sólo como posibles votantes y no como ciudadanos de derechos, les produce un distanciamiento con el tema.

5) Para finalizar y siendo coherente con una de las motivaciones de esta investigación, se insinuará un dialogo conceptual entre ciudadanía y participación, recordando que ciudadanía es un concepto epistemológico relacionado con el significado de ser sujeto social, mientras que participación constituye una operacionalización de la noción de ciudadanía, concepto orientado a lo práctico, lo político e influenciado por lo ideológico; frente a esta distinción se entenderá que la ciudadanía, desde el significado que hace sentido a los estudiantes ( sujetos), es aún un proceso incipiente que requiere una co- construcción colectiva, en forma integradora, para la formación desde una educación compleja. A la luz de ese vacío conceptual, la participación estudiantil, aún esta distante de una mirada socio-política para la llamada pedagogía realizadora que apunte al desarrollo humano, en elementos fundamentales como la confianza, la colaboración y la cultura de paz, que funden bases sólidas para una sociedad civil reflexiva, activa, dialogante y democrática. 


\section{REFERENCIAS}

Araneda, C., Neumann, N., Pedraja, L. y Rodriguez, E., Análisis exploratorio de las percepciones sobre los estilos de liderazgo, DOI: 10.4067/SO718-50062016000600013, Revista Formación Universitaria, 9(6) ,139$152(2016)$

Arendt, H., La Condición Humana, Editorial Paidós, Buenos Aires, Argentina (2009)

Baeza y otros, Representaciones sociales de la noción de ciudadanía en jóvenes de enseñanza media. Tesis para optar al título de Psicólogo, Universidad de Santiago de Chile, Facultad de Humanidades, Escuela de Psicología (2000)

Briede, J., Cabello, M., Pérez, C. y Arriagada, A., Plataforma Colaborativa para la Gestión de Proyectos de Diseño Industrial, DOI 10.4067/SO718-5006201600030008, Revista Formación Universitaria, 9(3), 61-74 (2016)

Bourdieu, P., Homo Academicus, Editorial Siglo XXI, Editores Argentina (2008)

Cabrera, F. Qué educación para qué ciudadanía. En Soriano Ayala, E. (Coord.) Interculturalidad: Fundamentos, Programas y Evaluación, Editorial La Muralla, Madrid (2002)

Cortina, A., Justicia Cordial, $1^{\circ}$ Ed., 29-64, Editorial Mínima TROTTA, Madrid (2010)

Costa, L., Barros V., Lopes M. y Marques, L., La formación docente y la educación de jóvenes y adultos. Análisis de la práctica pedagógica para la enseñanza de las ciencias. DOI 10.4067/SO71850062015000100002, Revista Formación Universitaria, 8(1) ,3-12(2015).

Cox, C., Políticas educacionales en el cambio de siglo. La reforma del sistema escolar en Chile. Editorial Universitaria, Santiago de Chile (2003)

Espejo, R., Interacción Simbólica en un acto de subjetividad de género. DOI:10.4067/SO71850062015000500006, Revista Formación Universitaria, 8(5), 43-58(2015)

Espíndola, J.L., Ética Ciudadana. Fundamentos, Editorial Porrúa, México (2009)

Francke, D. y Ojeda, P., Historiografía e historia de mujeres: estrategias para su inclusión en los procesos de enseñanza - aprendizaje en la educación media chilena, DOI: 10.4067/S0718-07052013000100021, Revista Estudios Pedagógicos, 39(1) ,361-375(2013)

García, M. y Conde, A., Liderazgo y calidad en las organizaciones educativas. En González J.M., García Guiu (Coord.). http://hdl.handle.net/10481/31240, ISBN: 978-84-338-5536-7, Líderes al servicio de la sociedad. Liderazgo en Entornos Internacionales, Universidad de Granada, 93-103 (2013)

Giroux, H.A., Placeres Inquietantes, 1르 Ed., 169-237, Paidós Educador, Barcelona (1996)

Giroux, H.A., Juventud Educación y Rol en la Integración en los Pueblos, Revista de CIECAS № 39, Editorial Mundo Siglo XXI, México (2015)

Gobierno de Chile, MINEDUC, Estudio Internacional de Educación Cívica y Formación Ciudadana, ICCS/IEA09, Chile (2010)

Gobierno de Chile, MINEDUC, Ley 20911 de Formación Ciudadana, Gobierno de Chile (2016)

Hernández, R., Fernández, C. y Baptista, P., Fundamentos de metodología de la investigación, 1aㅡ Ed., 155240. Mc Graw Hill, Interamericana Editores S.A., Madrid, España (2007)

Martínez Migueléz, M., Ciencia y Arte en la Metodología Cualitativa. 1aㅡ Ed., 137-180. Trillas México D.F., México (2004)

Muñoz, C. y Martínez R., Prácticas Pedagógicas y competencias ciudadanas. El caso del docente de historia de Chile. Doi: 10.15517/aje.v15i3.20658, Revista Actualidades Investigativas en Educación, 15(3), 1-22 (2015) 
Olivo, María G., Desarrollo Local, Política Social e Intervención Social, VII Coloquio Internacional de estudiantes de Trabajo Social "Desarrollo, Política Social e Intervención profesional, Universidad del Altiplano", (1-24), Puno, Perú, 8 al 10 de agosto (2007)

Programa de las Naciones Unidas para el Desarrollo, Desarrollo Humano en Chile, Más sociedad para gobernar el futuro. Santiago de Chile (2000)

Programa de las Naciones Unidas para el Desarrollo, Desarrollo Humano en Chile, Nosotros los chilenos: Un desafío cultural. Santiago de Chile (2002)

Programa de las Naciones Unidas para el desarrollo, La democracia en América hacia una democracia de ciudadanas y ciudadanos, $2^{a}$ Ed., Chile (2004)

Romeo, J., Los objetivos fundamentales transversales en busca de un currículo holístico, DOI: 10.4067/S0718-07052001000100009, Revista Estudios Pedagógicos, 1(27),119-130 (2001)

Rubio, J., Paradigmas de la Política, del Estado justo al estado legítimo. Editorial del Hombre Anthropos. Barcelona, España (1990)

Schütz, A., Luckmann, T., Las estructuras del mundo de la vida, $2^{\circ}$ Ed., Amorrortu Editores, 25-35, Buenos Aires, Argentina (2009)

Tomé, M., Berrocal, E. y Buendía, L., Contents for intercultural citizenship training. Student proposals after the first year of civic education (secondary school subject). An exploratory study, Revista de Investigación Educativa, Universidad de Murcia, 28(2), 425-444 (2010)

Váter, M., Nitschack, H., Hannah Arendt: Sobrevivir al totalitarismo. $1^{\circ}$ Ed., 103-138, Ediciones LOM, Santiago de Chile (2008)

Vilas, C., Deconstruyendo la Ciudadanía: Fragmentación Social, Globalización, Economía y Política de Identidades, Revista Universitaria Semestral, Estudios Sociales, Año IX, № 17, Santa Fe, Argentina, $2^{\circ}$ Semestre, págs. 111-132 (1999) 
